\title{
1,3,4-噁二唑三氮烯衍生物的合成及生物活性测定
}

\author{
雷 强 ${ }^{a}$ 秦上尚 ${ }^{b}$ 冯翠宁 ${ }^{a}$ 李佩佩 ${ }^{a}$ 张 茜 ${ }^{a}$ 龙 跃*, $a$ \\ $\left({ }^{a}\right.$ 郑州大学化学与分子工程学院 郑州 450001) \\ ( ${ }^{b}$ 郑州大学药学院 郑州 450001)
}

\begin{abstract}
摘要 利用拼合原理, 将具有生物活性的三氮烯结构与 1,3,4-噁二唑结构相拼合, 设计合成了 11 个未见报道的 $1,3,4-$ 噁二唑三氮烯衍生物. 所合成化合物经 ${ }^{1} \mathrm{H}$ NMR, IR 和 HRMS 得到表征. 用四甲基偶氮唑盐(MTT)法法评价了该类化 合物对胃癌细胞(MGC803)和前列腺癌细胞(PC-3)的抑制作用，结果显示化合物 2-[4-(3,3-二甲基三氮烯-1-基)苯 基]-5-(4-甲氧基苯基)-1,3,4-噁二唑 $\left(\mathbf{b}_{4}\right) 、 2$-[4-(3,3-二甲基三氮烯-1-基)苯基]-5-(2-甲氧基苯基)-1,3,4-噁二唑( $\mathbf{b}_{9}$ )、2-[4-(3,3二甲基三氮烯-1-基)苯基]-5-(3,4-甲叉二氧基苯基)-1,3,4-噁二唑( $\mathbf{b}_{10}$ )、2-[4-(3,3-二甲基三氮烯-1-基)苯基]-5-(吡啶-4基)-1,3,4-葰二唑 $\left(\mathbf{b}_{11}\right)$ 对前列腺癌细胞的抑制作用强于典型三氮烯药物达卡巴嗪(DTIC), 其 $\mathrm{IC}_{50}$ 值分别为 74.145, 87.790, 87.327 和 $104.875 \mu \mathrm{mol} / \mathrm{L}$, 而对胃癌细胞则几乎没有抑制作用. 采用微量肉汤稀释法测试了该类化合物对大肠埃希菌 (E. coli.)和金黄葡萄球菌(S. aureus)的抑制作用，结果显示这类化合物对这两种细菌并没有表现出抑制作用.
\end{abstract}

关键词 三氮烯; 1,3,4-惩二唑; 合成; 抗肿瘤活性；抑菌活性

\section{Synthesis and Biological Activities of 1,3,4-Oxadiazole Triazene Derivatives}

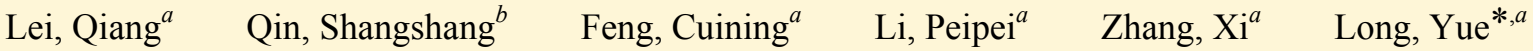 \\ ( ${ }^{a}$ College of Chemistry and Molecular Engineering, Zhengzhou University, Zhengzhou 450001) \\ ( ${ }^{b}$ School of Pharmaceutical Sciences, Zhengzhou University, Zhengzhou 450001)
}

\begin{abstract}
11 novel 1,3,4-oxadiazole triazene derivatives were designed and synthesized by combination of triazenes and 1,3,4-oxadiazoles. All the target compounds were characterized by ${ }^{1} \mathrm{H}$ NMR, IR and HRMS. The antitumor activities of all compounds were screened by using thiazolylblue (MTT) assay in vitro, in which MGC803 cell lines and PC-3 cell lines were used as the test cancer cell. The results showed that compounds 2-(4-(3,3-dimethyltriaz-1-en-1- yl)phenyl)-5-(4-methoxy-

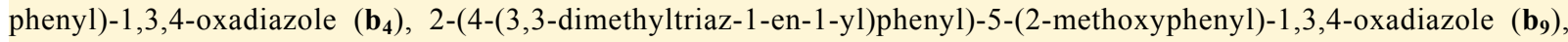
2-(4-(3,3-dimethyltriaz-1-en-1-yl)phenyl)-5-(benzo[d][1,3]dioxol-5-yl)-1,3,4-oxadiazole ( $\left.\mathbf{b}_{10}\right)$ and 2-(4-(3,3-dimethyltriaz-1en-1-yl)phenyl)-5-(pyridin-4-yl)-1,3,4-oxadiazole $\left(\mathbf{b}_{11}\right)$ exhibited higher activity against PC-3 cell than control compound dacarbazine (DTIC), and their $\mathrm{IC}_{50}$ values were $74.145,87.790,87.327$ and $104.875 \mu \mathrm{mol} / \mathrm{L}$, respectively. But all compounds had little inhibition effect on MGC803 cell lines. All synthesized compounds were screened by broth dilution technique for their fungicidal activity against $S$. aureus and E. coli. The antibacterial activity results showed that all of compounds were inactive.
\end{abstract}

Keywords triazenes; 1,3,4-oxadiazoles; synthesis; antitumor; antibacterial

三氮烯类化合物又称重氮氨基化合物，是指一类在 分子中含有 $\mathrm{N}=\mathrm{N}-\mathrm{N}$ 结构的化合物. 三氮烯类化合物 最广泛的应用是作为显色剂测定微量金属离子的浓度, 其优点是易于合成, 灵敏度高, 选择性好 ${ }^{[1,2]}$. 随着研究
的深入，人们逐渐发现，这类化合物还可以作为配体与 金属离子配位催化化学反应 ${ }^{[3,4]}$. 由于三氮烯分子中含 有三个共轭的氮原子, 这为合成含氮杂环类化合物提供 了基础 ${ }^{[5,6]}$. 此外，三氮烯结构中的重氮氨基，还可以提

*E-mail: longyue@zzu.edu.cn

Received July 3, 2015; revised October 7, 2015; published online October 26, 2015.

Project supported by the National Natural Science Foundation of China (No. J1210060), the Science and Technology Planning Project of Henan Province (No. 0624420031) and the Basic Research Development Program of Henan Province (No. 022463001).

国家自然科学基金(No. J1210060)、河南省科技攻关计划(No. 0624420031)、河南省基础研究基金(No. 022463001)资助项目. 
供高反应活性的芳基自由基或芳基阳离子 ${ }^{[7,8]}$, 形成不 同类型的碳碳键.

三氮烯类化合物在药物化学中的应用比较少见. 达 卡巴嗪(DTIC)和替莫唑胺(TMZ) 是仅有的两种应用于临 床的三氮烯类药物. 这两种药物临床用于治疗转移性黑 色素瘤、霍奇金淋巴瘤、非霍奇金淋巴瘤、软组织肉瘤 等肿瘤疾病 ${ }^{[9,10]}$. 有文献表明 ${ }^{[11]}$, 三氮烯类药物的体内 作用机理与 DNA 的 $O^{6}$-鸟嘌呤发生甲基化有关, 但其真 正的作用机理现在还不十分清楚. 同其他治疗肿瘤的烷 化剂一样, 三氮烯类药物也会生一些副作用如头痛、呕 吐、疲劳、便秘等 ${ }^{[12]}$.<smiles>CN(C)/N=N/c1[nH]cnc1C(N)=O</smiles>

DTIC<smiles></smiles>

TMZ
杂环类化合物通常具有许多生物活性. 1,3,4-噁二 唑是含有一个氧原子, 两个氮原子的五元芳香杂环化合 物, 其衍生物同样具有广泛的生物活性, 如抗癌、抑菌、 抗病毒等活性 ${ }^{[13 ~ 15]}$, 因此经常将其作为优势结构用于 药物分子的设计中.

为了设计一类新型具有抗癌活性的三氮烯类化合 物及开发三氮烯类药物新的抗癌活性, 我们将具有广泛 生物活性的 1,3,4-噁二唑结构与三氮烯结构进行拼合, 以使其活性相叠加, 得到对多种肿瘤细胞有较强抑制作
用，并具有类药性质的新型三氮烯类化合物.

\section{1 结果与讨论}

\section{1 合成}

以 4-氨基苯甲酸为原料, 经重氮化反应，与二甲胺 偶联，得到 4-(3,3-二甲基三氮烯-1-基)苯甲酸. 随后经 过酯化、肼解、与芳香醛缩合得到苯甲酰腙衍生物. 在 碘分子作用下, 环合得到 1,3,4-噁二唑类化合物(Scheme $1)$.

三氮烯的合成采用芳基重氮盐与二甲胺偶联的方 法. 在合成时应严格控制反应的温度及 $\mathrm{pH}$ 值, 以免造 成重氮盐的分解及副反应的增加. 由于 4-氨基苯甲酸含 有吸电子基团，使得其碱性较弱，难以溶解在盐酸中， 因此可以加入有机溶剂助溶，我们尝试了丙酮、DMSO、 DMF 和乙腈辅助溶解, 结果发现四种溶剂都可以得到 重氮盐, 但是乙腈的效果最好.

以 4-氨基苯甲酸为原料, 经多步反应得到苯甲酰 腙, 在碘分子作用下环合得到 1,3,4-啞二唑类化合物. 根据文献报道 ${ }^{[16]}$, 其机理如 Scheme 2 所示.

\section{2 结构表征}

所合成的化合物在红外光谱图中均有明显的特征 吸收. 三氮烯基团中 $\mathrm{N}=\mathrm{N}$ 的伸缩振动吸收峰在 1410 $1440 \mathrm{~cm}^{-1}, \mathrm{~N}-\mathrm{N}$ 的伸缩振动吸收峰在 $1120 \sim 1150$ $\mathrm{cm}^{-1}$. 1155 1250 $\mathrm{cm}^{-1}$ 为 $\mathrm{C}-\mathrm{O}-\mathrm{C}$ 的伸缩振动吸收峰, $1570 \sim 1650 \mathrm{~cm}^{-1}$ 为 $\mathrm{C}=\mathrm{N}$ 的伸缩振动吸收峰.

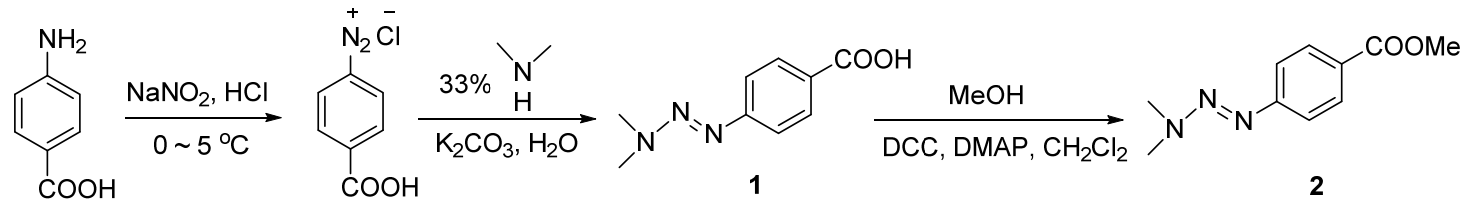

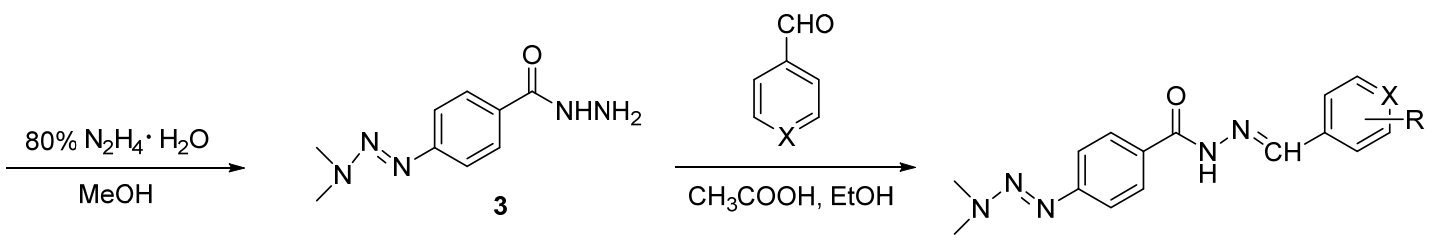<smiles>[R]c1ccccc1-c1nnc(-c2ccc(/N=N/N(C)C)cc2)o1</smiles>

$$
a_{1} \sim a_{11}
$$

$\mathbf{a}_{1}, \mathbf{b}_{\mathbf{1}}: \mathrm{X}=\mathrm{CH}, \mathrm{R}=4-\mathrm{OPh} ; \mathbf{a}_{\mathbf{2}}, \mathbf{b}_{\mathbf{2}}: \mathrm{X}=\mathrm{CH}, \mathrm{R}=2-\mathrm{F} ; \mathbf{a}_{\mathbf{3}}, \mathbf{b}_{\mathbf{3}}: \mathrm{X}=\mathrm{CH}, \mathrm{R}=3-\mathrm{CH}_{3} ; \mathbf{a}_{\mathbf{4}}, \mathbf{b}_{\mathbf{4}}: \mathrm{X}=\mathrm{CH}, \mathrm{R}=4-\mathrm{OCH}_{3} ;$ $\mathbf{a}_{5}, \mathbf{b}_{5}: \mathrm{X}=\mathrm{CH}, \mathrm{R}=4-\mathrm{N}\left(\mathrm{CH}_{3}\right)_{2} ; \mathbf{a}_{6}, \mathbf{b}_{6}: \mathrm{X}=\mathrm{CH}, \mathrm{R}=3,4-\left(\mathrm{OCH}_{3}\right)_{2} ; \mathbf{a}_{7}, \mathbf{b}_{7}: \mathrm{X}=\mathrm{CH}, \mathrm{R}=\mathrm{H} ; \mathbf{a}_{\mathbf{8}}, \mathbf{b}_{\mathbf{8}}: \mathrm{X}=\mathrm{CH}, \mathrm{R}=3-$ OEt; $\mathbf{a}_{\mathbf{9}}, \mathbf{b}_{\mathbf{9}}: \mathrm{X}=\mathrm{CH}, \mathrm{R}=2-\mathrm{OCH}_{3} ; \mathbf{a}_{\mathbf{1 0}}, \mathbf{b}_{10}: \mathrm{X}=\mathrm{CH}, \mathrm{R}=3,4-\mathrm{OCH}_{2} \mathrm{O} ; \mathbf{a}_{11}, \mathbf{b}_{11}: \mathrm{X}=\mathrm{N}, \mathrm{R}=\mathrm{H}$

图式 1 目标化合物的合成路线

Scheme 1 Synthesis of target compounds 


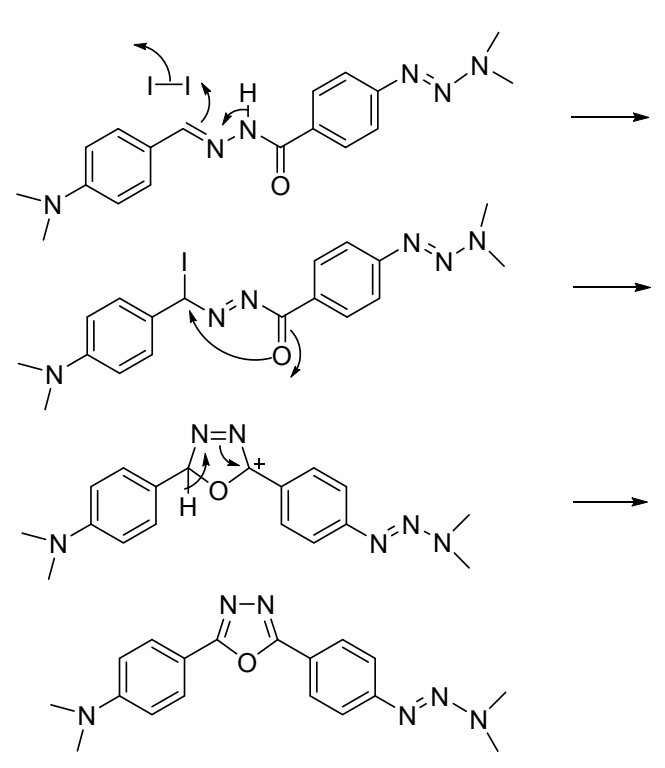

图式 2 1,3,4-惡二唑类化合物生成机理

Scheme 2 Proposed mechanism for the formation of 1,3,4-oxadiazoles.

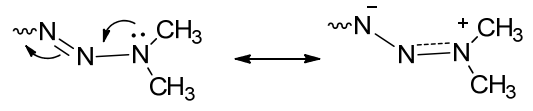

图式 3 末端氮原子的 $\mathrm{p}-\pi$ 共轭

Scheme 3 The $\mathrm{p}-\pi$ conjugation of terminal nitrogen atom

三氮烯基末端两个甲基的质子，为不等价质子. 造成两 个甲基不等价的原因是三氮烯基末端氮原子的孤对电 子与 $N=N$ 双键 $p-\pi$ 共轭, 使得末端 $N-N$ 单键具有双 键的性质而不能自由旋转, 导致 $\mathrm{N}$ 原子上两个甲基受到 分子内不同的屏蔽作用, 从而给出不同的质子信号.

\section{3 化合物的抗肿瘤活性}

我们以典型的三氮烯类药物达卡巴嗪(DTIC)为对 照, 测试了化合物 $\mathbf{b}_{1} \sim \mathbf{b}_{11}$ 对胃癌细胞(MGC803)和前列 腺癌细胞(PC-3)的抑制作用，其 $\mathrm{IC}_{50}$ 值见表 1 .

\section{4 化合物的抑菌活性}

三氮烯类化合物可以与蛋白质中含有 $\mathrm{n}$ 电子的羧 基、氨基、統基等基团发生烷基化, 使蛋白质正常的生 化反应受阻, 从而达到抑菌的作用. 但是三氮烯类化合 物作为一类烷化剂其抑菌作用研究较少.

我们以美罗培南(MEM)和利奈唑胺(LNZ)为对照, 测试了所合成化合物对大肠埃希菌 (E. coli.) 和金黄葡萄 球菌(S. aureus) 的抑制作用, 结果见表 2 .

\section{2 结论}

从其抗癌活性结果可以看出, 所合成的新型三氮烯 类化合物对 PC-3 细胞具有抑制作用, 而对 MGC803 细 胞几乎没有作用. 化合物 $\mathbf{b}_{\mathbf{4}}, \mathbf{b}_{\mathbf{9}}, \mathbf{b}_{\mathbf{1 0}}, \mathbf{b}_{\mathbf{1 1}}$ 对 PC-3 细胞的
表 1 化合物的抗肿瘤活性数据 $\left[\mathrm{IC}_{50} /\left(\mu \mathrm{mol} \cdot \mathrm{L}^{-1}\right)\right]$

Table 1 The anticancer activity of target compounds [ $\mathrm{IC}_{50}$ $\left.\left(\mu \mathrm{mol} \cdot \mathrm{L}^{-1}\right)\right]$

\begin{tabular}{ccc}
\hline 化合物 & PC-3 & MGC803 \\
\hline $\mathbf{b}_{\mathbf{1}}$ & 144.912 & - \\
$\mathbf{b}_{\mathbf{2}}$ & 180.040 & - \\
$\mathbf{b}_{\mathbf{3}}$ & 279.177 & - \\
$\mathbf{b}_{\mathbf{4}}$ & 74.145 & - \\
$\mathbf{b}_{\mathbf{5}}$ & 286.641 & - \\
$\mathbf{b}_{\mathbf{6}}$ & 112.153 & 299.718 \\
$\mathbf{b}_{\mathbf{7}}$ & 143.307 & - \\
$\mathbf{b}_{\mathbf{8}}$ & 429.199 & - \\
$\mathbf{b}_{\mathbf{9}}$ & 87.790 & - \\
$\mathbf{b}_{\mathbf{1 0}}$ & 87.327 & - \\
$\mathbf{b}_{\mathbf{1 1}}$ & 104.875 & - \\
DTIC & 105.873 & 53.687 \\
\hline
\end{tabular}<smiles>CC(O)C1C(=O)N2C(C(=O)O)=C(S[C@@H]3CNC[C@H]3C(=O)N(C)C)C(C)[C@H]12</smiles><smiles>CC(=O)NCC1CN(c2ccc(N3CCOCC3)c(F)c2)C(=O)O1</smiles>

表 2 化合物的杀菌活性数据( $\mathrm{MIC} / \mu \mathrm{g})$

Table 2 The antibacterial activity of target compounds $(\mathrm{MIC} / \mu \mathrm{g})$

\begin{tabular}{ccc}
\hline 化合物 & E. coli & S. aureus \\
\hline $\mathbf{b}_{\mathbf{1}}$ & $>32$ & $>32$ \\
$\mathbf{b}_{\mathbf{2}}$ & $>32$ & $>32$ \\
$\mathbf{b}_{\mathbf{3}}$ & $>32$ & $>32$ \\
$\mathbf{b}_{\mathbf{4}}$ & $>32$ & $>32$ \\
$\mathbf{b}_{\mathbf{5}}$ & $>32$ & $>32$ \\
$\mathbf{b}_{\mathbf{6}}$ & $>32$ & $>32$ \\
$\mathbf{b}_{\mathbf{7}}$ & $>32$ & $>32$ \\
$\mathbf{b}_{\mathbf{8}}$ & $>32$ & $>32$ \\
$\mathbf{b}_{\mathbf{9}}$ & $>32$ & $>32$ \\
$\mathbf{b}_{\mathbf{1 0}}$ & $>32$ & $>32$ \\
$\mathbf{b}_{\mathbf{1 1}}$ & $>32$ & $>32$ \\
MEM & 0.03 & - \\
LNZ & - & 2 \\
\hline
\end{tabular}

抑制作用强于对照药物达卡巴嗪. 其中化合物 $\mathbf{b}_{4}$ 表现 出最强的抑制作用. 当苯环上增加甲氧基时, 活性下降 $\left(\mathbf{b}_{4}\right.$ Vs. $\mathbf{b}_{6}$ 或 $\mathbf{b}_{9}$ Vs. $\left.\mathbf{b}_{6}\right)$; 比较化合物 $\mathbf{b}_{6}$ 和 $\mathbf{b}_{10}$, 我们发现将 $\mathbf{b}_{6}$ 的两个甲氧基用 3,4-亚甲基二氧基代替，其活性增加， 这可能是由于其构象受到了限制的缘故. 另外, 1,3,4-惡 二唑环的 5-位连接吡啶基, 其活性要高于连接苯基的活 性( $\mathbf{b}_{7}$ Vs. $\left.\mathbf{b}_{11}\right)$.

我们选择了两种典型的革兰氏阳性菌和革兰氏阴 性菌来进行化合物抑菌活性的测试，结果发现这类化合 物对细菌均没有表现出期望的抑制活性. 对于真菌的抑 
制的测试还在进行中.

\section{3 实验部分}

\section{1 仪器与试剂}

Bruker DPX 400/300 MHz 超导核磁共振仪(TMS 为 内标, $\mathrm{CDCl}_{3}$ 为溶剂); Bruker Vector 22 傅里叶变换红外 光谱仪, $\mathrm{KBr}$ 压片; Waters Q-TOF Micro 质谱测定仪; X-24 型数字显微熔点仪(温度计未校正), 北京泰克仪器 有限公司. 所用试剂均为市售分析纯, 使用前按常规方 法处理. 实验所用的肿瘤细胞: 胃癌细胞(MGC803)、前 列腺癌细胞(PC-3)由郑州大学药学院提供; 实验所用测 试菌株由郑州大学第一附属医院检验科提供.

\section{2 合成}

\subsubsection{4-(3,3-二甲基三氮烯-1-基)苯甲酸(1)的合成}

在圆底烧瓶中将 $2.0 \mathrm{~g}$ (14.6 mmol)对氨基苯甲酸溶 于 $40 \mathrm{~mL}$ 乙腈和 $20 \mathrm{~mL}$ 水中, 加入 $7 \mathrm{~mL}$ 浓盐酸, 置于 冰浴中搅拌. 将 $2.01 \mathrm{~g}(29.2 \mathrm{mmol})$ 亚硝酸钠溶于少量水 中, 缓慢滴加到圆底烧瓶中, 继续冰浴搅拌约 $2 \mathrm{~h}$, 得到 重氮盐。

另取一个圆底烧瓶加入 $33 \%$ 二甲胺溶液 $5.97 \mathrm{~g}$ (43.8 mmol), $6.04 \mathrm{~g}$ (43.8 mmol)碳酸钾以及少量的水, 冰浴搅拌下将重氮盐缓慢滴入. 滴加完毕后, 冰浴条件 下反应 3 4 h. 反应完毕后, 用稀盐酸调节 $\mathrm{pH}$ 值至 $2 \sim$ 3 , 乙酸乙酯萃取, 蒸干溶剂, 乙醇重结晶, 得到橘黄色 固体 $1.58 \mathrm{~g}$, 产率 56.1\%. m.p. $149 \sim 150{ }^{\circ} \mathrm{C} ;{ }^{1} \mathrm{H} \mathrm{NMR}$ $\left(400 \mathrm{MHz}, \mathrm{CDCl}_{3}\right) \delta: 7.91(\mathrm{~d}, J=8.8 \mathrm{~Hz}, 2 \mathrm{H}), 7.39$ (d, $J=$ $8.8 \mathrm{~Hz}, 2 \mathrm{H}), 3.53\left(\mathrm{~s}, 3 \mathrm{H}, \mathrm{NCH}_{3}\right), 3.18\left(\mathrm{~s}, 3 \mathrm{H}, \mathrm{NCH}_{3}\right)$.

3.2 .24 -(3,3-二甲基三氮烯-1-基)苯甲酸甲酯(2)的合 成

将 $1.58 \mathrm{~g}$ (8.19 mmol)化合物 $\mathbf{1}, 1.0 \mathrm{~g}(8.19 \mathrm{mmol})$ DMAP 溶于干燥的二氯甲烷中, 置于冰水浴中搅拌, 加 入 $1.68 \mathrm{~g}(8.19 \mathrm{mmol}) \mathrm{DCC}$, 继续搅拌 $30 \sim 40 \mathrm{~min}$, 加入 $1.57 \mathrm{~g}$ (49.1 mmol)无水甲醇，反应过夜. 反应完毕后， 加入适量的水继续搅拌, 使 DCC 吸水, 滤除沉淀后, 分 出有机相, 依次用碳酸氢钠、稀盐酸洗涤, 分出有机相, 干燥, 蒸出溶剂, 得黄色固体 $1.21 \mathrm{~g}$, 产率 71.3\%. m.p. 98 99 ${ }^{\circ} \mathrm{C} ;{ }^{1} \mathrm{H}$ NMR (400 MHz, $\left.\mathrm{CDCl}_{3}\right) \delta: 8.03$ (d, $J=8.8$ $\mathrm{Hz}, 2 \mathrm{H}), 7.47$ (d, $J=8.8 \mathrm{~Hz}, 2 \mathrm{H}), 3.92$ (s, 2H), 3.54 (s, 3H, $\left.\mathrm{NCH}_{3}\right), 3.26\left(\mathrm{~s}, 3 \mathrm{H}, \mathrm{NCH}_{3}\right)$.

3.2.3 4-(3,3-二甲基三氮烯-1-基)苯甲酰肼(3)和芳基 亚甲基-4-(3,3-二甲基三氮烯-1-基)苯甲酰腙 $\left(\mathbf{a}_{1} \sim \mathbf{a}_{11}\right)$ 的合成

化合物 3 合成方法参照文献[17]. 化合物 $\mathbf{a}_{1} \sim \mathbf{a}_{11}$ 合 成方法参照文献[18], 产物不经过分离纯化, 蒸除溶剂
后直接投入下步反应.

3.2.42-[4-(3,3-二甲基三氮烯-1-基)苯基]-5-芳基$1,3,4$-愳二唑 $\left(\mathbf{b}_{1} \sim \mathbf{b}_{11}\right)$ 的合成

化合物 $\mathbf{b}_{1} \sim \mathbf{b}_{11}$ 合成方法参照文献[16]. 将 0.45 $\mathrm{mmol}$ 三氮烯基苯甲酰腙化合物 $\mathbf{a}_{\mathbf{1}} \sim \mathbf{a}_{11}, 0.19 \mathrm{~g}$ (1.35 $\mathrm{mmol})$ 碳酸钾, $0.14 \mathrm{~g}(0.54 \mathrm{mmol})$ 碘, 置于圆底烧瓶中, 加入 $10 \mathrm{~mL} \mathrm{DMSO}, 100{ }^{\circ} \mathrm{C}$ 下摚拌反应, TLC 监测反应 结束, 加入适量的水, 乙酸乙酯萃取, 有机相分别用硫 代硫酸钠溶液, 饱和氯化钠溶液洗涤, 无水硫酸镁干燥, 柱层析分离 $[V$ (石油醚) : $V$ (乙酸乙酯 $)=3: 1]$.

2-[4-(3,3-二甲基三氮烯-1-基)苯基]-5-(4-苯氧基苯 基)-1,3,4-噁二唑 $\left(\mathbf{b}_{1}\right)$ ：淡黄色固体 $0.156 \mathrm{~g}$ ，产率 90.3\%. m.p. $153 \sim 154{ }^{\circ} \mathrm{C} ;{ }^{1} \mathrm{H}$ NMR $\left(400 \mathrm{MHz}, \mathrm{CDCl}_{3}\right) \delta: 8.10$ $(\mathrm{d}, J=6.8 \mathrm{~Hz}, 2 \mathrm{H}), 7.90(\mathrm{~d}, J=7.6 \mathrm{~Hz}, 1 \mathrm{H}), 7.80(\mathrm{~m}, 1 \mathrm{H})$, $7.59(\mathrm{~d}, J=6.8 \mathrm{~Hz}, 2 \mathrm{H}), 7.51(\mathrm{t}, J=2.4,8.0 \mathrm{~Hz}, 1 \mathrm{H})$, $7.39 \sim 7.43(\mathrm{~m}, 2 \mathrm{H}), 7.17 \sim 7.28(\mathrm{~m}, 2 \mathrm{H}), 7.10(\mathrm{~d}, J=8.8$ $\mathrm{Hz}, 2 \mathrm{H}), 3.58\left(\mathrm{~s}, 3 \mathrm{H}, \mathrm{NCH}_{3}\right), 3.28\left(\mathrm{~s}, 3 \mathrm{H}, \mathrm{NCH}_{3}\right)$; FT-IR $(\mathrm{KBr}) v: 3042,3011,1603(\mathrm{C}=\mathrm{N}), 1448(\mathrm{~N}=\mathrm{N}), 1227$ $(\mathrm{C}-\mathrm{O}-\mathrm{C}), 1160(\mathrm{~N}-\mathrm{N}) \mathrm{cm}^{-1}$; MS (ESI) $\mathrm{m} / \mathrm{z}: 386.2$ $[\mathrm{M}+\mathrm{H}]^{+}$; HRMS calcd for $\mathrm{C}_{22} \mathrm{H}_{20} \mathrm{~N}_{5} \mathrm{O}_{2}[\mathrm{M}+\mathrm{H}]^{+}$ 386.1617 , found 386.1615 .

2-[4-(3,3-二甲基三氮烯-1 - 基)苯基]-5-(2-氟苯 基)-1,3,4-噁二唑 $\left(\mathbf{b}_{2}\right)$ ：淡黄色固体 $0.125 \mathrm{~g}$, 产率 88.9\%. m.p. $129 \sim 130{ }^{\circ} \mathrm{C} ;{ }^{1} \mathrm{H}$ NMR (400 $\left.\mathrm{MHz}, \mathrm{CDCl}_{3}\right) \delta: 8.11 \sim$ $8.19(\mathrm{~m}, 3 \mathrm{H}), 7.53 \sim 7.59(\mathrm{~m}, 3 \mathrm{H}), 7.26 \sim 7.35(\mathrm{~m}, 2 \mathrm{H})$, $3.58\left(\mathrm{~s}, 3 \mathrm{H}, \mathrm{NCH}_{3}\right), 3.27\left(\mathrm{~s}, 3 \mathrm{H}, \mathrm{NCH}_{3}\right)$; FT-IR (KBr) v: 3062, 3040, 1653, $1612(\mathrm{C}=\mathrm{N}), 1453(\mathrm{~N}=\mathrm{N}), 1230$ $(\mathrm{C}-\mathrm{O}-\mathrm{C}), 1156(\mathrm{~N}-\mathrm{N}) \mathrm{cm}^{-1}$; MS (ESI) $\mathrm{m} / \mathrm{z}: 312.1$ $[\mathrm{M}+\mathrm{H}]^{+}$; HRMS calcd for $\mathrm{C}_{16} \mathrm{H}_{15} \mathrm{FN}_{5} \mathrm{O}[\mathrm{M}+\mathrm{H}]^{+}$ 312.1261, found 312.1263.

2-[4-(3,3-二甲基三氮烯-1-基)苯基]-5-(3-甲基苯 基)-1,3,4-啞二唑 $\left(\mathbf{b}_{3}\right)$ ：黄色固体 $0.132 \mathrm{~g}$, 产率 95.6\%. m.p. $121 \sim 122{ }^{\circ} \mathrm{C} ;{ }^{1} \mathrm{H}$ NMR (400 MHz, $\left.\mathrm{CDCl}_{3}\right) \delta: 8.11(\mathrm{~d}$, $J=8.6 \mathrm{~Hz}, 2 \mathrm{H}), 7.93 \sim 7.98(\mathrm{~m}, 2 \mathrm{H}), 7.58(\mathrm{~d}, J=8.6 \mathrm{~Hz}$, $2 \mathrm{H}), 7.34 \sim 7.45(\mathrm{~m}, 2 \mathrm{H}), 3.57\left(\mathrm{~s}, 3 \mathrm{H}, \mathrm{NCH}_{3}\right), 3.26(\mathrm{~s}, 3 \mathrm{H}$, $\left.\mathrm{NCH}_{3}\right), 2.47\left(\mathrm{~s}, 3 \mathrm{H}, \mathrm{CH}_{3}\right)$; FT-IR (KBr) v: 3050, 3021, 1655, $1627(\mathrm{C}=\mathrm{N}), 1454(\mathrm{~N}=\mathrm{N}), 1226(\mathrm{C}-\mathrm{O}-\mathrm{C}), 1160$ $(\mathrm{N}-\mathrm{N}) \mathrm{cm}^{-1}$; MS (ESI) $m / z: 308.2[\mathrm{M}+\mathrm{H}]^{+}$; HRMS calcd for $\mathrm{C}_{17} \mathrm{H}_{18} \mathrm{~N}_{5} \mathrm{O}[\mathrm{M}+\mathrm{H}]^{+}$308.1511, found 308.1509.

2-[4-(3,3-二甲基三氮烯-1-基)苯基]-5-(4-甲氧基苯 基)-1,3,4-噁二唑 $\left(\mathbf{b}_{4}\right)$ ：黄色固体 $0.141 \mathrm{~g}$. 产率 97.3\%. m.p. $189 \sim 190{ }^{\circ} \mathrm{C} ;{ }^{1} \mathrm{H}$ NMR $\left(300 \mathrm{MHz}, \mathrm{CDCl}_{3}\right) \delta: 8.10 \sim$ $8.07(\mathrm{~m}, 4 \mathrm{H}), 7.56$ (d, $J=8.7 \mathrm{~Hz}, 2 \mathrm{H}), 7.03$ (d, $J=9.0 \mathrm{~Hz}$, 2H), $3.90\left(\mathrm{~s}, 3 \mathrm{H}, \mathrm{OCH}_{3}\right), 3.54\left(\mathrm{~s}, 3 \mathrm{H}, \mathrm{NCH}_{3}\right), 3.29(\mathrm{~s}, 3 \mathrm{H}$, $\left.\mathrm{NCH}_{3}\right) ;{ }^{13} \mathrm{C}$ NMR 164.35, 164.20, 162.2, 153.41, 128.63, 
127.69, 120.97, 120.45, 116.64, 114.46, 55.47, 29.69. FT-IR (KBr) v: 3057, 2922, 1649, $1608(\mathrm{C}=\mathrm{N}), 1443$ $(\mathrm{N}=\mathrm{N}), 1250(\mathrm{C}-\mathrm{O}-\mathrm{C}), 1163(\mathrm{~N}-\mathrm{N}) \mathrm{cm}^{-1}$; MS (ESI) $m / z$ : $324.2[\mathrm{M}+\mathrm{H}]^{+}$; HRMS calcd for $\mathrm{C}_{17} \mathrm{H}_{18} \mathrm{~N}_{5} \mathrm{O}_{2}[\mathrm{M}+$ $\mathrm{H}]^{+}$324.1460, found 324.1457 .

2-[4-(3,3-二甲基三氮烯-1-基)苯基]-5-(4- $N, N$-二甲 基苯基)-1,3,4-噁二唑 $\left(\mathbf{b}_{5}\right)$ ：橘黄色固体 $0.131 \mathrm{~g}$, 产率 87.1\%. m.p. $189 \sim 190{ }^{\circ} \mathrm{C} ;{ }^{1} \mathrm{H}$ NMR (400 MHz, $\left.\mathrm{CDCl}_{3}\right) \delta$ : 8.10 (d, $J=8.8 \mathrm{~Hz}, 2 \mathrm{H}), 8.00$ (d, $J=8.8 \mathrm{~Hz}, 2 \mathrm{H}), 7.57$ (d, $J=8.4 \mathrm{~Hz}, 2 \mathrm{H}), 6.78(\mathrm{~d}, J=8.8 \mathrm{~Hz}, 2 \mathrm{H}), 3.56(\mathrm{~s}, 3 \mathrm{H}$, $\left.\mathrm{NCH}_{3}\right), 3.47$ (s, 3H, $\left.\mathrm{NCH}_{3}\right), 3.08$ (s, $6 \mathrm{H}, \mathrm{N}\left(\mathrm{CH}_{3}\right)_{2}$; FT-IR $(\mathrm{KBr}) v: 1607(\mathrm{C}=\mathrm{N}), 1439(\mathrm{~N}=\mathrm{N}), 1227(\mathrm{C}-\mathrm{O}-\mathrm{C})$, $1193(\mathrm{~N}-\mathrm{N}) \mathrm{cm}^{-1}$; MS (ESI) $m / z: 337.1[\mathrm{M}+\mathrm{H}]^{+}$; HRMS calcd for $\mathrm{C}_{18} \mathrm{H}_{21} \mathrm{~N}_{6} \mathrm{O}[\mathrm{M}+\mathrm{H}]^{+} 337.1777$, found 337.1774 .

2-[4-(3,3-二甲基三氮烯-1-基)苯基]-5-(3,4-二甲氧 基苯基)-1,3,4-噁二唑 $\left(\mathbf{b}_{6}\right)$ ：黄色固体 $0.150 \mathrm{~g}$, 产率 94.7\%. m.p. $185 \sim 186{ }^{\circ} \mathrm{C} ;{ }^{1} \mathrm{H}$ NMR $\left(300 \mathrm{MHz}, \mathrm{CDCl}_{3}\right) \delta$ : $8.10(\mathrm{~d}, J=6.75 \mathrm{~Hz}, 2 \mathrm{H}), 7.69(\mathrm{~m}, 2 \mathrm{H}), 7.57$ (m, 2H), 6.99 (d, $J=8.4 \mathrm{~Hz}, 1 \mathrm{H}), 4.01$ (s, $\left.3 \mathrm{H}, \mathrm{OCH}_{3}\right), 3.98$ (s, $3 \mathrm{H}$, OCH3), 3.54 (s, 3H, $\mathrm{NCH}_{3}$ ), 3.30 (s, 3H, $\mathrm{NCH}_{3}$ ); FT-IR $(\mathrm{KBr}) v: 3036,2912,1648,1607(\mathrm{C}=\mathrm{N}), 1443(\mathrm{~N}=\mathrm{N})$, $1229(\mathrm{C}-\mathrm{O}-\mathrm{C}), 1162(\mathrm{~N}-\mathrm{N}) \mathrm{cm}^{-1}$; MS (ESI) $\mathrm{m} / \mathrm{z}$ : $354.1[\mathrm{M}+\mathrm{H}]^{+}$; HRMS calcd for $\mathrm{C}_{18} \mathrm{H}_{20} \mathrm{~N}_{5} \mathrm{O}_{3}[\mathrm{M}+\mathrm{H}]^{+}$ 354.1566 , found 354.1563 .

2-[4-(3,3-二甲基三氮烯-1-基)苯基]-5-苯基-1,3,4-噁 二唑 $\left(\mathbf{b}_{7}\right)$ : 黄色固体 $0.119 \mathrm{~g}$, 产率 90.3\%. m.p. 161 $162{ }^{\circ} \mathrm{C} ;{ }^{1} \mathrm{H}$ NMR $\left(400 \mathrm{MHz}, \mathrm{CDCl}_{3}\right) \delta: 8.10 \sim 8.18(\mathrm{~m}$, $4 \mathrm{H}), 7.53 \sim 7.61(\mathrm{~m}, 5 \mathrm{H}), 3.58$ (s, $\left.3 \mathrm{H}, \mathrm{NCH}_{3}\right), 3.28(\mathrm{~s}, 3 \mathrm{H}$, $\left.\mathrm{NCH}_{3}\right)$; FT-IR $(\mathrm{KBr}) v: 1604(\mathrm{C}=\mathrm{N}), 1446(\mathrm{~N}=\mathrm{N}), 1210$ $(\mathrm{C}-\mathrm{O}-\mathrm{C}), 1156(\mathrm{~N}-\mathrm{N}) \mathrm{cm}^{-1}$; MS (ESI) $\mathrm{m} / z$ : 294.2 $[\mathrm{M}+\mathrm{H}]^{+}$; HRMS calcd for $\mathrm{C}_{16} \mathrm{H}_{16} \mathrm{~N}_{5} \mathrm{O}[\mathrm{M}+\mathrm{H}]^{+}$ 294.1355, found 294.1356.

2-[4-(3,3-二甲基三氮烯-1-基)苯基]-5-(3-乙氧基苯 基)-1,3,4-噁二唑( $\left.\mathbf{b}_{8}\right)$ : 黄色固体 $0.144 \mathrm{~g}$, 产率 94.7\%. m.p. $134 \sim 135{ }^{\circ} \mathrm{C} ;{ }^{1} \mathrm{H}$ NMR $\left(300 \mathrm{MHz}, \mathrm{CDCl}_{3}\right) \delta: 8.10$ $(\mathrm{d}, J=8.4 \mathrm{~Hz}, 2 \mathrm{H}), 7.66 \sim 7.23(\mathrm{~m}, 2 \mathrm{H}), 7.55 \sim 7.60(\mathrm{~m}$, 2H), $7.43(\mathrm{t}, J=7.8,8.1 \mathrm{~Hz}, 1 \mathrm{H}), 7.06 \sim 7.10(\mathrm{~m}, 1 \mathrm{H}), 4.15$ (dd, $J=6.9,7.2 \mathrm{~Hz}), 3.54$ (s, $\left.3 \mathrm{H}, \mathrm{NCH}_{3}\right), 3.29$ (s, $3 \mathrm{H}$, $\left.\mathrm{NCH}_{3}\right), 1.47$ (t, $J=7.2,6.9 \mathrm{~Hz}$ ); FT-IR $(\mathrm{KBr}) v: 3052$, 2977, 1683, $1604(\mathrm{C}=\mathrm{N}), 1446(\mathrm{~N}=\mathrm{N}), 1212(\mathrm{C}-\mathrm{O}-$ C), $1158(\mathrm{~N}-\mathrm{N}) \mathrm{cm}^{-1}$; MS (ESI) $m / z: 338.1[\mathrm{M}+\mathrm{H}]^{+}$; HRMS calcd for $\mathrm{C}_{18} \mathrm{H}_{20} \mathrm{~N}_{5} \mathrm{O}_{2}[\mathrm{M}+\mathrm{H}]^{+} 338.1617$, found 338.1619 .

2-[4-(3,3-二甲基三氮烯-1-基)苯基]-5-(2-甲氧基苯
基)-1,3,4-噁二唑( $\mathbf{b}_{\mathbf{g}}$ )：黄色固体 $0.133 \mathrm{~g}$, 产率 91.4\%. m.p. $105 \sim 106{ }^{\circ} \mathrm{C} ;{ }^{1} \mathrm{H}$ NMR $\left(300 \mathrm{MHz}, \mathrm{CDCl}_{3}\right) \delta: 8.10$ (d, $J=6.75 \mathrm{~Hz}, 2 \mathrm{H}), 8.00 \sim 8.03(\mathrm{~m}, 1 \mathrm{H}), 7.56(\mathrm{~d}, J=8.7$ $\mathrm{Hz}, 2 \mathrm{H}), 7.48 \sim 7.52(\mathrm{~m}, 1 \mathrm{H}), 7.06 \sim 7.13(\mathrm{~m}, 2 \mathrm{H}), 4.00(\mathrm{~s}$, $\left.3 \mathrm{H}, \mathrm{OCH}_{3}\right), 3.52\left(\mathrm{~s}, 3 \mathrm{H}, \mathrm{NCH}_{3}\right), 3.29\left(\mathrm{~s}, 3 \mathrm{H}, \mathrm{NCH}_{3}\right)$; FT-IR $(\mathrm{KBr}) v: 1605(\mathrm{C}=\mathrm{N}), 1445(\mathrm{~N}=\mathrm{N}), 1212(\mathrm{C}-$ $\mathrm{O}-\mathrm{C}), 1161(\mathrm{~N}-\mathrm{N}) \mathrm{cm}^{-1}$. MS (ESI) $\mathrm{m} / \mathrm{z}: 324.2[\mathrm{M}+$ $\mathrm{H}]^{+}$; HRMS calcd for $\mathrm{C}_{17} \mathrm{H}_{18} \mathrm{~N}_{5} \mathrm{O}_{2}[\mathrm{M}+\mathrm{H}]^{+} 324.1460$, found 324.1458 .

2-[4-(3,3-二甲基三氮烯-1-基)苯基]-5-(3,4-甲叉二 氧基苯基)-1,3,4-噁二唑 $\left(\mathbf{b}_{10}\right)$ ：黄色固体 $0.138 \mathrm{~g}$. 产率 90.9\%. m.p. $176 \sim 177{ }^{\circ} \mathrm{C}$; $\left.1 \mathrm{H} \mathrm{NMR} \mathrm{(400} \mathrm{MHz,} \mathrm{CDCl}_{3}\right) \delta$ : $8.09(\mathrm{~d}, J=6.8 \mathrm{~Hz}, 2 \mathrm{H}), 7.69(\mathrm{dd}, J=1.6,8.0 \mathrm{~Hz}, 1 \mathrm{H})$, $7.56 \sim 7.60(\mathrm{~m}, 3 \mathrm{H}), 6.96(\mathrm{~d}, J=8.0 \mathrm{~Hz}, 1 \mathrm{H}), 6.09(\mathrm{~s}, 2 \mathrm{H}$, $\mathrm{OCH}_{2} \mathrm{O}$ ), 3.58 (s, $\left.3 \mathrm{H}, \mathrm{NCH}_{3}\right), 3.27$ (s, $\left.3 \mathrm{H}, \mathrm{NCH}_{3}\right)$; FT-IR (KBr) $v$ : 3035, $1602(\mathrm{C}=\mathrm{N}), 1468(\mathrm{~N}=\mathrm{N}), 1233(\mathrm{C}-\mathrm{O}-$ C), $1157(\mathrm{~N}-\mathrm{N}) \mathrm{cm}^{-1}$; MS (ESI) $m / z: 338.2[\mathrm{M}+\mathrm{H}]^{+}$; HRMS calcd for $\mathrm{C}_{17} \mathrm{H}_{16} \mathrm{~N}_{5} \mathrm{O}_{3}[\mathrm{M}+\mathrm{H}]^{+} 338.1253$, found 338.1252 .

2-[4-(3,3-二甲基三氮烯-1-基)苯基]-5-(吡啶-4基)-1,3,4-噁二唑 $\left(\mathbf{b}_{11}\right)$ ：黄色固体 $0.129 \mathrm{~g}$, 产率 97.9\%. m.p. $226 \sim 227{ }^{\circ} \mathrm{C} ;{ }^{1} \mathrm{H}$ NMR (400 MHz, $\mathrm{CDCl}_{3}$ ) $\delta: 8.85$ (d, $J=6.0 \mathrm{~Hz}, 2 \mathrm{H}), 8.12$ (d, $J=8.4 \mathrm{~Hz}, 2 \mathrm{H}), 8.01(\mathrm{~m}, 2 \mathrm{H})$, 7.59 (d, $J=8.4 \mathrm{~Hz}, 2 \mathrm{H}), 3.59$ (s, $\left.3 \mathrm{H}, \mathrm{NCH}_{3}\right), 3.28$ (s, $3 \mathrm{H}$, $\left.\mathrm{NCH}_{3}\right)$; FT-IR $(\mathrm{KBr}) v$ : 3434, 3320, 1621, $1578(\mathrm{C}=\mathrm{N})$, $1397(\mathrm{~N}=\mathrm{N}), 1245(\mathrm{C}-\mathrm{O}-\mathrm{C}), 1160(\mathrm{~N}-\mathrm{N}) \mathrm{cm}^{-1}$; MS (ESI) $m / z: 295.1[\mathrm{M}+\mathrm{H}]^{+}$; HRMS calcd for $\mathrm{C}_{15} \mathrm{H}_{15} \mathrm{~N}_{6} \mathrm{O}$ $[\mathrm{M}+\mathrm{H}]^{+}$295.1307, found 295.1308.

\section{3 生物活性测试}

\subsection{1 抗癌活性测试}

所用的肿瘤细胞: 胃癌细胞(MGC803)、前列腺癌细 胞(PC-3). 实验细胞均用改良的 RPMI-1640 培养基(含 $10 \%$ 的胎牛血清和 $1 \%$ 的双抗) 在 $37{ }^{\circ} \mathrm{C}$ 的 $\mathrm{CO}_{2}$ 餒箱中培 养( $95 \%$ 的氧气, $5 \% \mathrm{CO}_{2}$, 保持在适当的湿度).

将培养血中的肿瘤细胞在培养箱中培养到一定数 量, 用计数板计数后, 配成一定浓度的细胞混悬液, 接 种于 96 孔培养板, 每孔加 $100 \mu \mathrm{L}$ 细胞混悬液. 在孵箱 培养 $24 \mathrm{~h}$, 待细胞贴壁后分别设阴性对照组、加药组, 阴性对照组设置 8 个复孔, 加药组设置 6 个浓度, 每个 浓度 4 个复孔. 将不同浓度的药物均匀混入 $200 \mu \mathrm{L}$ 培养 基中, 并分别加入各孔. 培养 $48 \mathrm{~h}$ 后向每孔中加入 5 $\mathrm{g} / \mathrm{L}$ 的四甲基偶氮唑盐(MTT)溶液 $20 \mu \mathrm{L} .4 \mathrm{~h}$ 后弃去上清 液, 每孔加入二甲亚砜 (DMSO) $150 \mu \mathrm{L}$, 置于摇床摇 10 $\min$ 使结晶物溶解. 在酶标仪上测其吸光度值 $(A, \lambda=490$ $\mathrm{nm})$, 结果以复孔 $A$ 的均值表示. 对测得的数值进行处 
理, 分别计算出阴性对照组和各个加药组的标准差、平 均值和加药组的存活率. 做存活率的柱状图观察不同浓 度药物作用结果的梯度情况. 利用 SPSS 软件进行线性 回归求出每个药物的 $\mathrm{IC}_{50}$.

\subsection{2 抑菌活性测试}

采用美国临床实验室标准委员会(Clinical and Laboratory Standards Institute, CLSI) M07-A9 标准推荐的 微量肉汤稀释法 ${ }^{[19]}$, 分别测定了 11 种化合物对大肠埃 希菌 ATCC25922 和金黄色葡萄球菌 ATCC29213 的最低 抑菌浓度(MICs). 具体方法如下: 按照倍比稀释原则, 将抗菌药物原液用水解酪蛋白肉汤 $(\mathrm{MH})$ 稀释成 10 个梯 度, 依次加入 96 孔细胞培养板前 10 个孔中, 每孔 100 $\mu \mathrm{L}$. 用 $\mathrm{MH}$ 肉汤将测试菌株稀释成 $105 \mathrm{CFU} / \mathrm{mL}$, 在每 排前 10 孔各加入稀释菌液 $100 \mu \mathrm{L}$. 第 11 孔加 $100 \mu \mathrm{L}$ $\mathrm{MH}$ 肉汤作阴性对照, 第 12 孔加 $100 \mu \mathrm{L}$ 稀释菌液作阳 性对照. $37{ }^{\circ} \mathrm{C}$ 培养 16 20 h, 观察各孔培养液中细菌的 生长情况, 以抑制细菌生长的药物最高稀释度作为测试 药物的最小抑菌浓度(MIC). 每种药物做 2 个平行. 参比 药物: 美罗培南(大肠埃希菌)和利奈唑胺 (金黄色葡萄球 菌), 参比药物判定结果参照 CLSI M100-S22 (2012)标 准 ${ }^{[19]}$, 美罗培南 $(\mathrm{S}: \leqslant 1 \mu \mathrm{g} / \mathrm{mL}, \mathrm{I}: 2 \mu \mathrm{g} / \mathrm{mL}, \mathrm{R}: \geqslant 4$ $\mu \mathrm{g} / \mathrm{mL})$, 利奈唑胺 $(\mathrm{S}: \leqslant 4 \mu \mathrm{g} / \mathrm{mL}, \mathrm{R}: \geqslant 8 \mu \mathrm{g} / \mathrm{mL})$. 达到 或高于参比药物 MIC 16 倍以内认为化合物具有抗菌活 性, 进而扩大受试菌株评价数目 $(n \geqslant 10)$, 评价 $\mathrm{MIC}_{50}$ 和 $\mathrm{MIC}_{90}$.

辅助材料(Supporting Information) 所有新化合物的 ${ }^{1} \mathrm{H}$ NMR, IR 和HRMS谱图. 这些材料可以免费从本刊网 站(http://sioc-journal.cn/)上下载.

\section{References}

[1] Zhang, X. H.; Chen, W. B. Asian J. Chem. 2013, 25, 9805.
[2] Zhu, C. S.; Chen, W. B. Asian J. Chem. 2013, 25, 7409.

[3] Chu, J.; Xie, X. H.; Yang, S. R.; Zhan, S. Z. Inorg. Chim. Acta 2014, 410, 191.

[4] Stefan, B.; Stefan, D.; Frank, L.;Nicholas, E. L.; Emma, L. S. Bioorg. Med. Chem. Lett. 2002, 12, 1849.

[5] Sun, H.; Wang, C. M.; Yang, Y. F.; Chen. P.; Wu, Y. D.; Zhang, X H.; Huang, Y. J. Org. Chem. 2014, 79, 11863.

[6] Koji, T.; Mohammad A. A.; Naoyuki, H.; Johan, W.; Hiroshi, S.; Yoshitsugu, S.; Hayato, F.; Satoshi, S.; Mitsuhiro, A. J. Org. Chem. 2014, 79, 6366.

[7] Nan, G. M.; Zhou, J. Chin. J. Org. Chem. 2012, 32, 1695 (in Chinese). (南光明, 周均, 有机化学, 2012, 32, 1695.)

[8] Zhou, J.; Yang, W. J.; Wang, B. J.; Ren, H. J. Angew. Chem., Int. Ed. 2012, 51, 12293.

[9] Simona, C.; Sabrina, F.; Giuseppe, S.; Maria, T. R.; Enzo, B.; Josef, J.; Stefania, D. A. Mol. Pharmacol. 2004, 66, 478.

[10] Reid, J. M.; Kuffel, M. J.; Miller, J. K.; Rios, R.; Ames, M. M. Clin. Cancer Res. 1999, 5, 2192

[11] Marchesi, F.; Turriziani, M.; Tortorelli, G.; Awisati, G.; Torino, F.; Vecchis, L. D. Pharmacol. Res. 2007, 56, 275.

[12] Yuichi, H.; Mitchel, S. B.; Russell, O. P. Cancer Res. 2001, 61, 1957

[13] Cledualdo, S. O.; Bruno, F. L.; Jose, M. B. F.; Jorge, G. F. L.; Petronio, F. A. F. Molecules 2012, 17, 10192.

[14] Liu, J. C.; Wang, W. D.; He, H. W. Chin. J. Org. Chem. 2014, 34, 1447 (in Chinese).

(刘建超, 王卫东, 贺红武, 有机化学, 2014, 34, 1447.)

[15] Sun, N. B.; Tong, J. Y.; Wu, H. K. Chin. J. Org. Chem. 2013, 33, 101 (in Chinese).

(孙娜波, 童建颖, 武宏科, 有机化学, 2013, 33, 101.)

[16] Yu, W. Q.; Huang, G.; Zhang, Y. T.; Liu, H. X.; Dong, L. H.; Yu, X. J.; Li, Y. J.; Chang, J. B. J. Org. Chem. 2013, 78, 10337.

[17] Gao, Y. L.; Lin, X. F.; Han, F. F.; Bao, X. P. Chin. J. Org. Chem. 2011, 31, 1648 (in Chinese). (高元否, 林选福, 韩菲菲, 鲍小平, 有机化学, 2011, 31, 1648.)

[18] Xu, Y.; Lei, P.; Ling, Y.; Wang, S. W.; Yang, X. L. Chin. J. Org. Chem. 2014, 34, 1118 (in Chinese). (徐炎, 雷鹏, 凌云, 王圣文, 杨新玲, 有机化学, 2014, 34, 1118.)

[19] Clinical and Laboratory Standards Institute. 2012. Performance standards for antimicrobial susceptibility testing; 20th informational supplement. M100-S22. Clinical and Laboratory Standards Institute, Wayne, PA. 